\title{
CORRECTION
}

Open Access

\section{Correction to: Unusual injury pattern (cauterization)}

Mohammed Madadin $^{{ }^{*}}$ and Khamis Almazrooei ${ }^{2}$

\section{Correction to: Egypt J Forensic Sci \\ https://doi.org/10.1186/s41935-018-0110-6}

The original version of the aforementioned article (Madadin and Almazrooei 2019) contained an error with regard to the institutional affiliation of the first author (Mohammed Madadin). The affiliation of the first author is corrected to "Department of Pathology, Imam Abdulrahman Bin Faisal University, Dammam, Saudi Arabia".

The authors, hereby, publish this correction note to be considered for all purposes.

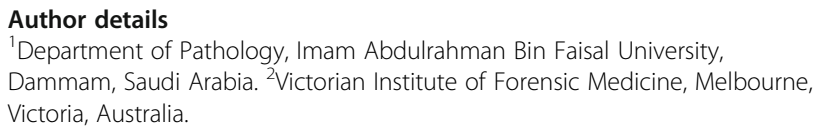

Published online: 24 October 2019

\section{Reference}

Madadin M, Almazrooei K (2019) Unusual injury pattern (cauterization). Egypt J

Forensic Sci 9:5. https://doi.org/10.1186/s41935-018-0110-6

\footnotetext{
* Correspondence: mmadadin@iau.edu.sa

The original article can be found online at https://doi.org/10.1186/s41935018-0110-6

'Department of Pathology, Imam Abdulrahman Bin Faisal University,

Dammam, Saudi Arabia

Full list of author information is available at the end of the article
} 